\title{
Research on Preventing Moral Hazard of Construction Project Based on Information Asymmetries
}

\author{
Pengcheng Xiang ${ }^{1,2, *}$ and Jinan Wang ${ }^{1}$ \\ ${ }^{1}$ Faculty of Construction Management and Real Estate, Chongqing University; ${ }^{2}$ Research Center of Construction \\ Economics and Management, Chongqing University, Chongqing, P.R. China
}

\begin{abstract}
There exists the problem of information asymmetry among the participants in construction project who form economic partnerships one another. Information asymmetries among the participants in construction project places a premium on adverse selection and moral hazard. The major objective of this article is to implement the mechanisms of incentive and monitoring under the framework of principal-agent theory in analysis of moral hazard of construction project and to explore how to prevent it. The optimization model of incentive and monitoring under the circumstance of asymmetric information will be founded on the basis of the analysis of the effect of incentive and monitoring mechanisms in the principal-agent relationship. It indicates that reliability of information can be increased when bringing incentive and monitoring mechanisms into reward contract, which can prevent moral hazard of construction project.
\end{abstract}

Keywords: Construction project risk, information asymmetries, moral hazard, preventing risk, principal-agent theory.

\section{INTRODUCTION}

From the perspective of information economics, opportunistic behavior induced by information asymmetry which means adverse selection \& moral hazard problem is the root causes of project risks. Adverse selection problem occurs for the information asymmetry happened before contract; the problem of moral hazard exists for the information asymmetry occurred after the contract. In the theory of adverse selection, the client signed the contract without knowing the type of agent, so the problem of the client is actually what kind of contract to choose to get the agent's private information. In the moral hazard theory, when a client signed a contract, he had known the type of the agent. However, after contracts, he cannot observe the action of agents, so the client's problem is to design an optimal incentive mechanism to induce the agent to choose the desired action of the client. In the construction market, there is asymmetric information among the owners (the client), the contractors and supervision units (the agent) from the beginning, which leads to the emergence of adverse selection and moral hazard. Moral hazard of project is due to the disagreement on maximization goals of clients and agents, after the client signed the principal-agent contract with the agent, the agent often use their information advantage to pursue their own interest maximization in project management activities, thus do something not conducive to commission and cause loss to the client.

In recent years, the problem of the moral hazard of construction project has attracted the attention of domestic and foreign scholars. Sha et al. (2004) analyzed the principalagent relationship and problems between participating

*Address correspondence to this author at the NO.174, Shazheng Street, Shapingba District, Chongqing, P.R. China. Postcard: 400045;

Tel: 13883689728; E-mail: pcxiang @cqu.edu.cn subjects from the information economics, and they thought that the main reason for the project risk is the information asymmetry which induces the opportunism behavior, namely the adverse selection and moral hazard cause the anomie of project participants' subject behavior [1]. Zhu et al. (2005) pointed out that there exists principal-agent relationship between the owner and the contractor in engineering construction and that the information asymmetry between them leads to the existence of moral hazard during the period of the construction .He built the moral risk model of contractor to analysis quantitatively how to establish the constraint mechanism to realize interest maximization of owners [2]. Wang et al. (2008) took use of incomplete information game theory to analyze the phenomenon that the low price wins the bid in the construction market. It shows that the construction enterprise in the drive of profit may bring moral risk of poor quality in the construction [3]. Schieg (2008) proposed that a project consists of many important project participants \& a large number of contracts and that the asymmetric distribution of information will cause adverse selection and moral hazard before and after the execution of the contract. He putted forward specific strategies to prevent asymmetric information based on the principal-agent theory in project management [4]. Bergmann (2008) proposed an optimal incentive contract to solve the problem of moral risk \& information asymmetry existing in the project [5]. Bond et al. (2009) thought its limited energy and different abilities of bearing risk can lead to poor efficiency of the allocation of the project resource and put forward the optimal incentive contract through the analysis of agent under the relationship of many tasks entrusted agent after studying the problem of many tasks in principal-agent [6]. Winch (2010) analyzed the reason for the producing moral risk in the projects and putted forward the relevant measures based on the principal-agent theory, including designing incentive mechanism in the contract, the price adjustment mechanism, the conflict resolution 
mechanism and so on [7]. Zhang (2011) set up an analysis model for moral hazard of construction project visual organization under various situations, obtain contract conditions to prevent moral hazard by solving the model, and prevent moral hazard by controlling contract condition parameters [8]. Arce (2012) discussed the adverse selection and moral hazard in principal agent theory, shows that it is possible to successfully screen for non-opportunistic agents who will exert more effort and accept less incentivized compensation as compared to opportunistic agents [9]. Wang et al. (2012) introduced a method to reduce information asymmetries by design optimal incentive mechanism for complementary special knowledge-sharing [10]. Xiang et al. (2012) explored risk prevention mechanisms and measures in construction projects on the basis of asymmetric information theory [11]. Fagart et al. (2013) applied for first order approach in characterizing the optimal contract, when the others focused on a particular version of the problem [12].

From related literatures, the problem of the moral risk has already become the main reason for the project risks caused by information asymmetry. Related literatures research focus on the causes of the moral risk and measures to solve to the moral risk problems from different angles come up. These researches pay much attention to qualitative analysis in instead of quantitative analysis; the proposed measures are too wide and lacking of pertinence \& corresponding theoretical support. This paper tries to break through the limitation of research in moral hazard of construction project. Incentive and monitoring mechanism is introduced into the prevention of moral hazard of construction project under the framework of the principal-agent theory and the optimal incentive and monitoring model is set up in the case of the information asymmetry. This paper discussed the prevention system of construction project moral hazard as to manage the moral hazard of construction project scientifically.

\section{RESEARCH METHODOLOGIES AND MODEL}

\subsection{The Fundamental Analysis Framework of Principal- agent Theory}

In the principal-agent relationship, the client would like the agent to choose operation in accordance with the interests of clients, but clients can not directly observe what actions the agent choose, and what they can observe is only some other variables, these variables are decided by the action of agents and other exogenous random factors together, therefore it is incomplete information for agents. Clients will reward or punish agents in accordance with those observed incomplete information in order to motivate them choose the most favorable action for clients.

Action combination of the agent can be indicated by A, $a \in A$ indicates a specific action of the agent. A can be decision variables of any dimension, for the simple problem analysis, it is assumed that $a$ is a one-dimensional variable about effort level of the agent. And $\theta$ is an exogenous random variable out the control of the agent, $G(\theta)$ and $G(\theta)$ are the distribution function and density function of $\theta$ in its value range respectively. After the agent to choose action $a$, $a$ and the exogenous variables $\theta$ jointly decide an observable outcome $x(a, \theta)$ of an output $R(a, \theta)$. It is assumed that
$\mathrm{R}$ is a strictly increasing concave function, that is, if $\theta$ is given, the harder the agent works, the higher the output will be, but the marginal productivity of efforts decreases; $\mathrm{R}$ is a strictly increasing function of $\theta$, that is, the higher $\theta$ represents more favorable natural conditions. At this point, the client can design an incentive contract $S(x)$ to reward or punish the agent according to the observed results $x$.

It is assumed that the expected utility functions of clients and agents are $V(R-S(x))$ and $U(S(R))-c(a)$ respectively under the condition that $V^{\prime}>0, V^{\prime \prime} \leq 0 ; U^{\prime}>0, U^{\prime \prime} \leq 0$; $c^{\prime}>0, c^{\prime \prime}>0$. This means that both clients and agents are risk averters or risk-neutral persons, and negative marginal effect of effort is increasing. The interests conflict of clients and agents comes from the assumptions that

$$
\frac{\partial R}{\partial a}>0 \text { and } c^{\prime}>0 \text { firstly, } \frac{\partial R}{\partial a}>0
$$

means that the client would like agents to make greater efforts, while $c^{\prime}>0$ predicates that the agents like less effort. Therefore, unless the client can provide the agent sufficient incentive, the agent will not work hard as the client hoped.

Client's expected utility function can be expressed as:

$$
\int V(R(a, \theta)-S(x(a, \theta))) g(\theta) d \theta \quad(P) \text {. }
$$

The client should choose $a$ and $S(x)$ to maximize the expected utility function, but the client is faced with two constraints from agents. The first constraint is the participation constraint, that is, the utility that the agent get from acceptance of the contract cannot be less than the greatest expected utility without acceptance of the contract $\bar{U}$ (reservation utility), this can be expressed as:

$$
\int U(s(x(a, \theta))) g(\theta)-c(a) \geq \bar{U} \quad(I R) .
$$

The second constraint is the incentive compatibility constraint, that is, it is given that the client agent can not observe the action a and nature state $\theta$, in any of the incentive contract, the agents always choose action a that can maximize their expected utility, so any expected a of a client can only achieve through utility maximization act of agents, it can be expressed as:

$$
\int U(s(x(a, \theta))) g(\theta)-c(a) \geq \int U\left(s\left(x\left(a^{\prime}, \theta\right)\right)\right) g(\theta) d \theta-c\left(a^{\prime}\right), \forall a^{\prime} \in A
$$

The client's problem is to choose $a$ and $S(x)$ to maximize the expected utility function $(\mathrm{P})$, which can satisfy the constraints (IR) and (IC) [13].

\subsection{The Optimal Incentive Model Under Asymmetric Information}

In the construction market, it is assumed that the principal (the owners) cannot observe the act choice $a$ of the agent (contractor or supervision units) and exogenous variables $\boldsymbol{\theta}$; they can only observe the output R. At this point, the agent's incentive compatibility constraint is working, because no matter how the client rewards or punishes the agent, agents will always choose the actions which can maximize their own utilities. Therefore, the client cannot use compulsory contract to force the agent to choose actions that the princi- 
pal hoped, but only through the incentive contract to maximize their expected utility function [14].

\subsubsection{The Basic Model of Optimal Incentive}

When owners design the incentive contracts, it is assumed that the agent (contractor or supervision units) has two kinds of actions in the course of project implementation, one is work hard $(\mathrm{H})$, the other is not work hard $(\mathrm{L})$, the project outputs is $R \in\left[R_{1}, R_{2}\right]$. When agents work hard, the distribution function and the distribution density of $R$ are $F_{H}(R)$ and $f_{H}(R)$ respectively; if the agent does not work hard, the distribution function and the distribution density of $R$ are $F_{L}(R)$ and $f_{L}(R)$ respectively. It is assumed that $R(a, \theta)$ is an increasing function of a, that is, the harder agents work, the higher the output will be. It takes $\mathrm{R}$ as a random variable, for $R \in\left[R_{1}, R_{2}\right]$, there is $F_{H}(R) \leq F_{L}(R)$, which indicates that the probability of obtaining high profits if agents work hard is greater than that when they does not work hard.

It is assumed that the principal (the owners) hopes the agents (contractors or supervision units) to choose working hard, the incentive contract is $S(R)$, the agent's incentive compatibility constraint is $\partial S / \partial R \neq 0$. In order to make the agent have sufficient positivity to work hard, the client must give up Pareto optimal risk-sharing contract. Therefore, the client access to the expected utility function as follows:

$$
V(R-S(R)) f_{H}(R) d R \text {. }
$$

When the clients maximize their own utility, they will be faced with two constraints of the agent. The first one is the participation constraint, which can be expressed as

$$
\int U(S(R)) f_{H}(R) d R-C(H) \geq \bar{U} ;
$$

the second constraint is the incentive compatibility constraint, which is expressed as:

$$
\int U(S(R)) f_{H}(R) d R-C(H) \geq \int U(S(R)) f_{L}(R) d R-C(L)
$$

To sum up, the problem of the principal (the owners) is to choose incentive contract $S(R)$ and solve the following optimization problem:

$$
\begin{aligned}
& \max _{S(R)} \int V(R-S(R)) f_{H}(T) d R \\
& S \bullet T \quad(I R) \quad \int U(S(R)) f_{H}(R) d R-C(H) \geq \bar{U} \\
& (I C) \quad \int U(S(R)) f_{H}(R) d R-C(H) \geq \int U(S(R)) f_{L}(R) d R-C(L)
\end{aligned}
$$

If $\lambda$ and $\mu$ are Lagrange multipliers involved in participant constraint IR and incentive compatibility constraint IC respectively, then the first-order conditions of the above optimization problem are as follows:

$-V^{\prime} f_{H}(R)+\lambda U^{\prime} f_{H}(R)+\mu U^{\prime} f_{H}(R)-\mu U^{\prime} f_{L}(R)=0$

Arrange formula (1), so

$$
\frac{V^{\prime}}{U^{\prime}}=\lambda+\mu\left(1-\frac{f_{L}(R)}{f_{H}(R)}\right)\left(\frac{f_{l}(R)}{f_{H}(R)}\right.
$$

is called likelihood ratio.)

When $\mu=0$, we can get Pareto-optimal risk-sharing conditions

$$
\frac{V^{\prime}}{U^{\prime}}=\lambda
$$

this happens to be the Pareto optimal risk-sharing of agent and client under asymmetric information, and when $\mu=0$, the incentive compatibility constraint is broken. Therefore, the optimal contract under asymmetric information is different from the optimal contract under symmetric information. When $\mu>0$, if the principal is risk neutral person, the Pareto optimal risk-sharing means that the agent does not bear any risk under symmetric information condition; in the case of information asymmetry, the agent must assume certain risks. It is assumed that incentive contract under asymmetric information is $S_{1}(R)$, in case of information asymmetry the incentive contract is $S(T)$. Then, if

$$
\begin{aligned}
& f_{L}(R) \geq f_{H}(R), \text { so } S(R) \leq S_{1}(R) \\
& \text { if } f_{L}(R)<f_{H}(R), \text { so } S(R)>S_{1}(R) .
\end{aligned}
$$

Likelihood ratio

$$
\frac{f_{l}(R)}{f_{H}(R)}
$$

is ratio that the given occurrence probability of $\mathrm{R}$ when the agent do not choose to work hard and the given occurrence probability of $\mathrm{R}$ when the agent choose to work hard, which indicates how much of $\mathrm{R}$ that the client observed is from the distribution $f_{L}(R)$. If the likelihood ratio

$$
\frac{f_{l}(R)}{f_{H}(R)}
$$

is higher, it means that $\mathrm{R}$ is more likely from the distribution $f_{L}(R)$; When

$$
\frac{f_{l}(R)}{f_{H}(R)}=1
$$

the possibility that $\mathrm{R}$ comes from distribution $f_{L}(R)$ is the same with that from distribution $f_{H}(R)$, so the client can not get new information. The client will infer from $\mathrm{R}$ that they observed that the agent has chosen to work hard or sleep, so as to reward or punish the agent. If they inferred the possibility that the client does not work hard is much bigger, they will punish the agent, so

$$
S(R)<S_{1}(R)
$$

if they think the agent is more likely to have choose working hard, they will reward the agent, so

$$
S(R)>S_{1}(R) \text {. }
$$

\subsubsection{The Instance Model of Optimal Incentives}

In the construction market, it is assumed that the achievement of the project management objectives depends on the level of efforts of the agent (contractor or supervision units) $a$ and exogenous variables $\theta$. It is assumed that the project outputs is a linear function form: $R=a+\theta$, where a which means the efforts of agents is one-dimensional variable, $\theta$, the exogenous variables, is a normally distributed 
random variable that its average is zero, variance is $\sigma^{2}$. Therefore, $E(R)=E(a+\theta)=a, \operatorname{Var}(R)=\sigma^{2}$ that is, the efforts degree of an agent can decide the average of output, but it does not affect the variance of output.

It is assumed that the principal is a risk neutral person, and the agent is a risk averter. The linear contract: $S(R)=\lambda+\mu R$ is considered, where $\lambda$ is the agent's fixed income, $\mu$ is the share of output of agents, that is, while outputs $\mathrm{R}$ increases a unit, the remuneration of agents increases $\mu$ units. $\mu=1$ means that agents bear all the risks, $\mu=0$ means that agents do not bear any risk. As the principal is risk neutral, given $S(R)=\lambda+\mu R$, the client's expected utility equals to the expected income:

$E V(R-S(R)=E(R-\lambda-\mu R)=-\lambda+E(1-\mu) R=-\lambda+(1-\mu) a$

It is assumed that utility function of the agent has the unchanging characteristics of the absolute risk aversion, the measure for absolute risk aversion is $\rho$, the agent's effort cost is $C(a)=\frac{b}{2} a^{2}$ (b is the cost factor, the greater $\mathrm{b}$ is, the greater the negative effect brought by the same efforts degree $a$ is). The real income of the agent is as follows:

$U=S(R)-C(a)=\lambda+\mu R-\frac{b}{2} a^{2}=\lambda+\mu(a+\theta)-\frac{b}{2} a^{2}$

The risk cost of the agent is $\frac{\rho}{2} \mu^{2} \sigma^{2}, E(U)$ is the agent's
cected revenue, there is: $E(U)-\frac{1}{2} \rho \mu^{2} \sigma^{2}=\lambda+\mu a-\frac{1}{2} \rho \mu^{2} \sigma^{2}-\frac{1}{2} b a^{2}$

It is said that $U$ is the reserved income level of an agent, then if the expected income of the agent is less than $\vec{U}$, agents will not accept the contract, the agent's participation constraint can be expressed as:

$\lambda+\mu a-\frac{1}{2} \rho \mu^{2} \sigma^{2}-\frac{1}{2} b a^{2} \geq \bar{U}$

When the client can observe the agent's effort degree a, the incentive constraint (IC) does not work, any level of a can be achieved through the mandatory contract meeting the participation constraint (IR). Therefore, the client's problem is to solve the following optimization problem:

$$
\begin{aligned}
& \max _{\lambda, \mu, a} E V=-\lambda+(1-\mu) a \\
& S \bullet T \quad(I R) \quad \lambda+\mu a-\frac{1}{2} \rho \mu^{2} \sigma^{2}-\frac{1}{2} b a^{2} \geq \bar{U}
\end{aligned}
$$

In optimal circumstances, the participation constraint is established, we can substitute participation constraint $\lambda$ into the objective function, there is:

$$
\max _{\lambda, \mu, a} E V=a-\frac{1}{2} \rho \mu^{2} \sigma^{2}-\frac{1}{2} b a^{2}-\bar{U}
$$

Calculate a derivation of $\mathrm{a}$,

$$
\frac{\partial E V}{\partial a}=1-b a
$$

assume

$$
\frac{\partial E V}{\partial a}=0
$$

so

$$
a=\frac{1}{b}, \mu=0
$$

substitute a and $\mu$ into the participation constraint, so

$$
\lambda=\bar{U}+\frac{1}{2 b} \text {. }
$$

As the principal is risk neutral, the agent is a risk averter, the Pareto optimal risk-sharing require that the agent does not bear any risk, that is $\mu=0$, at this time, the fixed income that the client pays to the agent coincides with the sum of the reservation utility of the agent and their effort cost.

When the client cannot observe the agent's level of effort a, given $\mu=0$, agents will choose $a$ to maximize their real incomes, that is

$$
\max _{\mu}=\lambda+\mu a-\frac{1}{2} \rho \mu^{2} \sigma^{2}-\frac{1}{2} b a^{2} .
$$

The first-order conditions of this optimization problem are as follows:

$$
a=\frac{\mu}{b}=0 \text {. }
$$

This indicates that if the agent's income has nothing to do with the output, the agent will choose $a=0$ instead of $a=\frac{1}{b}$

When the agent's efforts a can not be observed, given $\lambda$ and $\mu$, the incentive constraint for agents is

$$
a=\frac{\mu}{b},
$$

the problem of clients at this time is to solve the following optimization problem:

$$
\begin{aligned}
& \max _{\lambda, \mu} E V=-\lambda+(1-\mu) a \\
& S \bullet T \quad(I R) \quad \lambda+\mu a-\frac{1}{2} \rho \mu^{2} \sigma^{2}-\frac{1}{2} b a^{2} \geq \bar{U} \\
& \text { (IC) } a=\frac{\mu}{b}
\end{aligned}
$$

Substitute the participation constraint (IR) and incentive constraint (IC) into the objective function, the above optimization problem becomes:

$$
\max _{\lambda, \mu} E V=\frac{\mu}{b}-\frac{1}{2} \rho \mu^{2} \sigma^{2}-\frac{\mu^{2}}{2 b}-\bar{U}
$$

Calculate a derivation of $\mu$, so

$$
\frac{\partial E V}{\partial \mu}=\frac{1}{b}-\rho \mu \sigma^{2}-\frac{\mu}{b},
$$


given

$$
\frac{\partial E V}{\partial \mu}=0
$$

then

$$
\mu=\frac{1}{1+b \rho \sigma^{2}} \quad \mu=\frac{1}{1+b \rho \sigma^{2}}>0
$$

shows that the agent must take risks. If agents are riskneutral, that is $\rho=0$, then the optimal contract requires agents to assume full risk, that is $\mu=1 \cdot \mu$ is a decreasing function of $b 、 \rho 、 \sigma^{2}$, which shows that the greater agents avert risks, and afraid of hard work, the less risks they will assume.

Calculate derivatives of

$$
\rho \text {. } \sigma^{2} \text { in } \mu=\frac{1}{1+b \rho \sigma^{2}}
$$

respectively, there are:

$$
\frac{\partial \mu}{\partial \rho}=\frac{-1}{\left(1+b \rho \sigma^{2}\right)^{2}} 、 \frac{\partial \mu}{\partial \sigma^{2}}=\frac{-1}{\left(1+b \rho \sigma^{2}\right)^{2}} .
$$

As a result of $\frac{\partial \mu}{\partial \rho}<0$

And

$$
\frac{\partial \mu}{\partial \sigma^{2}}<0
$$

the optimal incentive contract should strike a balance between incentives and risk, if given $\mu$, the bigger $\rho$ or $\sigma^{2}$ is, the higher the risk cost will be, at this time the smaller the optimal risk-sharing will be.

\subsection{The Optimal Incentive \& Monitoring Model under Asymmetric Information}

In the principal-agent relationship, in order to prevent and avoid risks, in addition to the introduction of incentive mechanism design into optimal incentive contract, it is also very important to introduce monitoring mechanisms into the contract design [15-16].

In the construction market, the achievement of project management goals not only rely on the efforts degree of agent (contractor or supervision units) (a) and exogenous variables $(\theta)$, but also related to the monitoring measures of clients (owners) (S). Assumed that the project outputs is a linear function form: $R=a+\theta$, where the efforts degree of agents(a) is one-dimensional variable, the exogenous varia$\operatorname{bles}(\theta)$ is a normally distributed random variable of zero mean, and variance $\sigma^{2}, \sigma^{2}$ reflects the risk of exogenous variables; the monitoring measures of clients $\mathrm{S}$ is related to the effort degree of agents, control measures is the linear form: $S=a+\varepsilon$, where $\varepsilon$ is a normally distributed random variable of zero mean, and variance $\gamma^{2}, \gamma^{2}$ reflects the accuracy of surveillance and control measures. $\Theta$ and $\mathcal{E}$ are independent of each other, so the covariance $\operatorname{Cov}(\theta, \varepsilon)=0$.
From the analysis of 3, we can see that if it is assumed that the principal is risk neutral, the agent's remuneration shall be the fixed income, fixed compensation contract does not have stimulation, and clients bear the entire risk. If we consider stimulation and monitoring measures, remuneration function made by the principal also depends on the output $\mathrm{R}$ and control measures $\mathrm{S}$, assuming that the remuneration function is linear, so the compensation function is as follows:

$$
T(R, S)=F+\mu R+\lambda S=F+\mu(a+\theta)+\lambda(a+\varepsilon)
$$

Where $\mathrm{F}$ is the fixed remuneration of agents, $\mu$ is the share of agents to share the output $(0 \leq \mu \leq 1), \lambda$ is the reward paid to the agent based on the monitoring.

Assumed that the cost of the agent's efforts is

$$
C(a)=\frac{b}{2} a^{2}
$$

so the real income of the agent is as follows:

$$
W=T(R, S)-C(a)=F+\mu(a+\theta)+\lambda(a+\varepsilon)-\frac{1}{2} b a^{2}
$$

As the output $\mathrm{R}$ and control measures $\mathrm{S}$ has uncertainty, so the real income of the agent $\mathrm{W}$ is also uncertain, excluding uncertainties, the certain quantity of the income that the agent can get is the difference between the expected income and the risk premium, risk premium is expressed as half of the product of risk aversion degree $\rho$ and the variance $\operatorname{Var}(W)$. In that case, the agent's certainty equivalent income they can receive is as follows:

$$
U=E(W)-\frac{1}{2} \rho \operatorname{Var}(W)
$$

As

$$
\begin{aligned}
& E(W)=F+a(\mu+\lambda)-\frac{1}{2} b a^{2}, \\
& \operatorname{Var}(W)=\mu^{2} \sigma^{2}+\lambda^{2} \gamma^{2}, \text { so: } \\
& U=E(W)-\frac{1}{2} \rho \operatorname{Var}(W)=F+a(\mu+\lambda)-\frac{1}{2} b a^{2}-\frac{\rho}{2}\left(\mu^{2} \sigma^{2}+\lambda^{2} \gamma^{2}\right)
\end{aligned}
$$

Calculate a derivation of a in $\mathrm{U}$ :

$$
\begin{aligned}
& \frac{\partial U}{\partial a}=\mu+\lambda-b a \\
& \text { given } \frac{\partial U}{\partial a}=0
\end{aligned}
$$

the first order optimal conditions of $U$ are as follows:

$$
a=\frac{\mu+\lambda}{b} \text {. }
$$

According to the agent's participation constraint $U \geq \bar{U}$, we can see that the minimum fixed remuneration required by the agent for receiving the remuneration contract is as follows:

$$
F=\bar{U}-\frac{(\mu+\lambda)^{2}}{2 b}+\frac{\rho}{2}\left(\mu^{2} \sigma^{2}+\lambda^{2} \gamma^{2}\right)
$$


Assume that the principal is risks neutral, the income received by the client is as follows: $V=E(R-T(R, S))$, consider $R=a+\theta$ and formula (6), we can see:

$V=E(R-T(R, S))=E(a+\theta)-E(F+\mu(a+\theta)+\lambda(1+\gamma)=a-F-a(\mu+\lambda)$

Substitute

$$
a=\frac{\mu+\lambda}{b}
$$

and formula (9) into formula (10), so

$V=\frac{\mu+\lambda}{b}-\bar{U}+\frac{(\mu+\lambda)^{2}}{2 b}-\frac{\rho}{2}\left(\mu^{2} \sigma^{2}+\lambda^{2} \gamma^{2}\right)-\frac{(\mu+\lambda)^{2}}{b}=\frac{\mu+\lambda}{b}-\bar{U}-\frac{(\mu+\lambda)^{2}}{2 b}-\frac{\rho}{2}\left(\mu^{2} \sigma^{2}+\lambda^{2} \gamma^{2}\right)$

calculate derivation of $\lambda$ and $\mu$ in (11) respectively, the optimal first-order conditions of (11) are as follows:

$$
\begin{aligned}
& \frac{\partial V}{\partial \mu}=\frac{1}{b}-\frac{\mu+\lambda}{b}-\rho \mu \sigma^{2}=0 \\
& \text { that is } \\
& \mu=\frac{1-\lambda}{1+b \rho \sigma^{2}} \\
& \frac{\partial V}{\partial \lambda}=\frac{1}{b}-\frac{\mu+\lambda}{b}-\rho \lambda \gamma^{2}=0
\end{aligned}
$$

that is : $\lambda=\frac{1-\mu}{1+b \rho \gamma^{2}}$

Unite (12) and (13), we can see

$$
\begin{gathered}
\mu=\frac{\gamma^{2}}{\sigma^{2}+\gamma^{2}+b \rho \sigma^{2} \gamma^{2}} \\
\lambda=\frac{\sigma^{2}}{\sigma^{2}+\gamma^{2}+b \rho \sigma^{2} \gamma^{2}}
\end{gathered}
$$

\section{THE MODEL ANALYSIS AND RESULT}

According to the optimal incentive \& monitoring model under asymmetric information, we can conclude as following:

1) In the contract it is helpful to introduce incentive mechanism in order to improve the reliability of information transferred by agent. In the case of information asymmetry, the specific actions of agent cannot be observed by client so agent may completely first promise his action and deviate from the action in practice .For the asymmetric information is full of value, agent would deliver their own private information to the client only when agents get the value, which makes the degree of trust between the principal and agent very limited, this relationship of limited mutual trust also makes credibility that the principal pay remuneration to agent according to the distributor's efforts reduced. But the selection of agent campaign depends on the reward contract the principal provided, and if reward contract introduce incentive mechanism, the decision of agent will be induced by the reward contract. The designed contract just gets its practical significance only if principal induce the action of the agent by the contract. In the design of the contract, the principal usually compensate for the agent for not acquiring pri- vate information through motivational pattern of sharing the risk \& profit or induce the behavior of the agent through adding additional conditions to contracts, so that authenticity of the information passed by the agent in the performance of the contract increases in order to reduce the loss caused by information asymmetry.

2) The design which introduces incentive mechanism and monitoring mechanism into the remuneration contracts increases the degree of dependence on information. Clients can take a variety of ways to monitor, if they monitor the work time of the agent, $\mu$ can be understood as the compensation based on the output, $\lambda$ is the compensation paid for the work time. With the improvement of the monitor signal's accuracy, $\gamma^{2}$ will become smaller, we can infer from formula (14) that $\lambda$ will increase, based on (12) and (13), we can see that when $\lambda$ increased, the agent's share of output $\mu$ will reduce, which indicates that with the improvement of the monitor signal's accuracy, the percentage of payment based on the monitor signal will increase. When $\gamma^{2} \rightarrow 0$, that is, the workforce time can be almost precisely observed, then $\mu \rightarrow 0$ 、 $\lambda \rightarrow 1$, according to (6), the design of compensation contract should be based on the information provided by the monitor signal. Similarly, when $\gamma^{2} \rightarrow \infty$, that is, it is impossible to obtain monitor information through the method of monitoring, then $\mu \rightarrow 1 、 \lambda \rightarrow 0$, at this time, the design of compensation contract should be based on the information provided by the output. It can be seen that (12), (13) and (14) organically describes the relationship between incentives mechanisms and monitoring mechanisms in the design of remuneration contract. By (12) and (13), we can see that with the increase of $\lambda, \mu$ will reduce, or vice versa, it indicates that the share of output and monitor signals are interrelated in the enacting of remuneration contracts. In the extreme cases that $\lambda \rightarrow 0$, the share of output $\mu$ is only determined to the risk aversion degree of the agent and the size of the environment risk, and when $\lambda \rightarrow 1$, the share of output $\mu$ is 0 , that is the remuneration contract is only based on the monitoring information.

3) There is nothing to do with the degree of agent's efforts and fixed compensation paid by the principal. We can see from

$$
a=\frac{\mu+\lambda}{b}
$$

that in project management the efforts degree of agent (contractor or supervision units) has nothing to do with a fixed remuneration paid for them. From the client (owner) point of view, regardless of whether the fixed compensation the client paid is high or low will not have an impact on the efforts of agents, which suggests that the system of fixed remuneration has no incentives function to agents. When $\mu$ and $\lambda$ are zero, $a=0$, which indicates that if there is neither incentive system nor monitor system, the extent of the agent's efforts is zero. This is because the agent has no incentive to work harder without incentive system; no control mechanism means that there is no pressure to force agents to work harder. Although the fixed remuneration system does not have incentive 
function, but it does not mean that there is no need to fix the remuneration in contract design. We can see from (9) that only the agent's fixed remuneration equals to (9) at least, the agents is willing to accept the payment program provided by the client, otherwise the client cannot reach an agreement with the agent.

4) The control measures clients take will have a negative impact on agents. When the client introduces control mechanisms, it may result in effectiveness loss to the agent, which may lead the agent to refuse to cooperate with the client. Faced with this situation, the client may be required to pay compensation to the agent for the effectiveness loss caused by monitor measures, for example, the client can increase the minimum reserved utility $U$ or additional subsidies, thus increasing the attractiveness to the agent, at the same time it could cause the agent motivated to reveal information about his efforts to prove that he should get a minimum reserved utility or the additional subsidies, thereby reduce the situation of information asymmetry in the principal-agent relations [17].

\section{CONCLUSION AND FUTURE}

The relationship between the principal (owners) and agent (contractor and supervisor) is essentially a kind of principal-agent cooperative relationship based on the contract. In order to complete the cooperation, all parties are asked to provide each other information, take risk, and get paid and so on. When they pursuit their own interest maximization, the contractor and supervisor tend to get their own interest maximization at the sacrifice of the owner's interest, which can lead to the conflict of interest of both sides. In order to balance the conflict of interest, a series of contract is needed to develop to achieve the goal of all parties [18]. Whether these contracts are effective and make the interest of all parties maximum depends on whether the information the parties own is symmetric when the owner, the contractor and the supervisor conclude, perform and evaluate contract. The interests \& cooperation of principal \& agent depend on not only the effort of both sides but also some exogenous variables. Although in some extent the client could know the probability distribution of the exogenous variables, but he could not exactly know the kind of what actually happened, which provide the agent opportunity to hide efforts. In construction market, when the goal of the project is not met, the owner is not sure the reason is whether the contractor or supervisor units do not work hard or exogenous risk happened. In the face of such environment of uncertainty, the owner not only can not judge the degree of the attempts of contractor or supervisor but also not sure how much total utility is created by the effort of the contractor or supervisor. Therefore, the client need tie the pay to the degree of the attempts contractor and supervisor make, but the degree of efforts is their private information which can take him benefit. Therefore, in the face of the conflicts of interest produced by information asymmetry and the moral risk of agents, the owner must take incentive and restraint mechanisms to solve they. Incentive and monitoring mechanism make dynamic balance of the distribution of interests \&accountability of both sides under the condition of the information asymmetry as to achieve consistency of the goal of both sides whose information is different. Bringing the incentive and monitoring mechanism into the design of appointment contract and paying remuneration can improve the reliability of information agent passes, which is import to handle the conflicts of interest between principal and agent to reach the purpose of preventing moral hazard of construction project.

In practice, it has important function to take the incentive and monitoring mechanism into the project contract for preventing moral hazard of construction project, but the key to the problem lies in the oversight of the contract. The contract will transfer part of risk from owner to the contractor to let the contractor perceive risk response, and the result of the response is that the contractor work hard. The contract includes incentives terms \&monitoring terms and sets the solution to the future uncertainty. Owing to the influence of the factors such as uncertainty, information asymmetry and information cost, the terms of the monitoring and motivate contract is not complete in itself, it is uncertain to actually execute the terms of the contract. Therefore, there is need to perfect and improve the corresponding laws \&regulations and set effective mechanism to execute contract and manage risk $[19,20]$. This is precisely the problem that needs further discussion and research.

\section{CONFLICT OF INTEREST}

The authors confirm that this article content has no conflict of interest.

\section{ACKNOWLEDGEMENTS}

The work described in this paper was fully supported by a joint grant from Supported by Chongqing Social Science Foundation Project (2013ZDZZ02), Project No. CDJKXB13004, 106112012CDJSK5503 supported by the Fundamental Research Funds for the Central Universities. Any opinions, findings, and conclusions or recommendations expressed in this material are those of the authors.

\section{REFERENCES}

[1] K.X. Sha, Q. Song, J.Z. Zhao, et al. "See the rectifying and standardizing the construction market through asymmetric information", Construction Economics, no. 1, pp. 82-85, 2004.

[2] B. Zhu, and Q. M. Li, "Moral hazard model analysis in the construction period under asymmetric information", Journal of Chongqing Construction University, vol. 27, no. 4, pp. 102-105, 2005.

[3] W. G. Wang, and D. H. Liu, "The analysis of incomplete information game theory in construction project about the low bidding phenomenon", Management Science of China, vol. 16, pp. 444 449, 2008

[4] M. Shier, "Strategies for avoiding asymmetric information in construction project management", Journal of Business Economics and Management, vol. 9. no.1, pp. 47-51, 2008.

[5] R. Bergmann, and G. Fried, "Controlling innovative projects with moral hazard and asymmetric information", Research Policy, vol. 37, no. 9, pp. 1504-1514, 2008.

[6] P. Bond, and A. Gomes, "Multitask principal-agent problems: Optimal contracts, fragility, and effort misallocation", Journal of Economic Theory, vol. 144, no.1, pp. 175-211, 2009.

[7] G. M. Winch, "Managing Construction Projects", Wiley-Blackwell, 2010.

[8] E. Y. Zhang, "On moral hazard of construction project visual organization”, Proscenia Engineering, no, 12, pp. 156-162, 2011.

[9] G. Daniel, "Acre Principals' preferences for agents with social preferences", Journal of Economic Behavior and Organization, vol. 90, pp. 154-163, 2013. 
[10] M.Z. Wang, C.Y.C. Sha, "Special knowledge sharing incentive mechanism for two clients with complementary knowledge: A principal-agent perspective", Expert Systems with Applications, vol. 39, no.3, pp. 3153-3161, 2012.

[11] P. C. Xiang, J. Zhou, and X. Y. Zhou, "Construction Project Risk Management Based on the View of Asymmetric Information", Journal of Construction Engineering and Management, vol. 138, no.11, pp. 1303-1311, 2012.

[12] M. Fagart, and C. Flute, "The first-order approach when the cost of effort is money", Journal of Mathematical Economics, vol. 49, no. 1, pp. 7-16, 2013.

[13] A. R. Stephen, "The Economic Theory of Agency: The Principal's Problem", American Economic Review, vol. 63, no. 2, pp. 134-139, 1973.

[14] R. J. Arnott, J. E. Stiglitz, "The Basic Analytics of Moral Hazard", Scandinavian Journal of Economics, vol. 90, no. 3, pp. 383-413, 1988.

[15] X.H. Dang, X. F. Quan, and M.L. Yang, "The monitoring and incentive game analysis in risk investment agency relationships", Research Management, vol. 28, no. 1, pp. 155-170, 2007.
[16] Y.L. Xu, Z.Y. Qi, D. Liu, "The design and expansion of incentive mechanism framework model based on monitoring mechanisms", Journal of Ha Erbin gongye university, vol. 38,vol. 10pp. 162616292006.

[17] Y. Xiang, and H. Ren, "The analysis of incentive and monitoring mechanism about project Supervision under asymmetric information", Journal of Chongqing Construction University, vol. 27, no.6, pp. 113-116, 2005.

[18] J. J. Liu, and J. F. Liang, "The analysis of optimal entrusted financing contract under moral hazard", Journal of Systems Engineering, vol. 24, no.5, pp. 602-606, 2009.

[19] W. M. Di, M. J. Wang, and J.R. Liu, "The discussion of construction project management mechanisms based on moral hazard prevention", Journal of Xi'an Technology University, vol. 24, no.4, pp. 522-526, 2004.

[20] P. C. Xiang, M. D. Ou, and Y. Chen, "The management methods of integrated risk project based on the theory of asymmetric information", Science and Technology Management Research, vol. 29, no.5, pp. 516-518, 2009.

Received: September 16, 2014

Revised: December 23, 2014

Accepted: December 31, 2014

(C) Xiang and Wang; Licensee Bentham Open.

This is an open access article licensed under the terms of the Creative Commons Attribution Non-Commercial License (http://creativecommons.org/licenses/by-nc/3.0/) which permits unrestricted, non-commercial use, distribution and reproduction in any medium, provided the work is properly cited. 